\title{
Enhancing the specificity and efficiency of polymerase chain reaction using polyethyleneimine-based derivatives and hybrid nanocomposites
}

\author{
This article was published in the following Dove Press journal: \\ International Journal of Nanomedicine \\ 21 February 2012 \\ Number of times this article has been viewed
}

\author{
Weiwei Tong ${ }^{1,2}$ \\ Xueyan $\mathrm{Cao}^{2}$ \\ Shihui Wen ${ }^{2}$ \\ Rui Guo² \\ Mingwu Shen ${ }^{2}$ \\ Jianhua Wang ${ }^{3}$ \\ Xiangyang Shi ${ }^{1,2,4}$
}

'State Key Laboratory for Modification of Chemical Fibers and Polymer Materials, Donghua University, Shanghai, ${ }^{2}$ College of Chemistry, Chemical Engineering and Biotechnology, Donghua University, Shanghai, ${ }^{3}$ Department of Biochemistry and Molecular Cell Biology, School of Medicine, Shanghai Jiao Tong University, Shanghai, People's Republic of China; ${ }^{4}$ Centro de Química da Madeira, Universidade da Madeira, Funchal, Portugal

Correspondence: Xiangyang Shi 2999 North Renmin Road, College of Chemistry, Chemical Engineering and Biotechnology, Donghua University, Shanghai 201620, People's Republic of China

Tel +86 2I 67792656

Fax +86 2I 67792306804

Email xshi@dhu.edu.cn

Jianhua Wang

Department of Biochemistry and

Molecular Cell Biology, Shanghai Jiao Tong

University School of Medicine, Shanghai

200025, People's Republic of China

Tel +86 2l 5466087|

Fax +8621 63842I57

Email jianhuaw2007@gmail.com
Abstract: There is a general necessity to improve the specificity and efficiency of the polymerase chain reaction (PCR), and exploring the PCR-enhancing mechanism still remains a great challenge. In this paper we report the use of branched polyethyleneimine (PEI)-based derivatives and hybrid nanocomposites as a novel class of enhancers to improve the specificity and efficiency of a nonspecific PCR system. We show that the surface-charge polarity of PEI and PEI derivatives plays a major role in their effectiveness to enhance the PCR. Positively charged amine-terminated pristine PEI, partially $(50 \%)$ acetylated PEI (PEI-Ac $\mathrm{c}_{50}$ ), and completely acetylated PEI (PEIAc) are able to improve PCR efficiency and specificity with an optimum concentration order of PEI $<$ PEI-Ac ${ }_{50}<$ PEI-Ac, whereas negatively charged carboxyl-terminated PEI (PEI-SAH; SAH denotes succinamic acid groups) and neutralized PEI modified with both polyethylene glycol (PEG) and acetyl (Ac) groups (PEI-PEG-Ac) are unable to improve PCR specificity and efficiency even at concentrations three orders of magnitude higher than that of PEI. Our data clearly suggests that the PCR-enhancing effect is primarily based on the interaction between the PCR components and the PEI derivatives, where electrostatic interaction plays a major role in concentrating the PCR components locally on the backbones of the branched PEI. In addition, multiwalled carbon nanotubes modified with PEI and PEI-stabilized gold nanoparticles are also able to improve the PCR specificity and efficiency with an optimum PEI concentration less than that of the PEI alone, indicating that the inorganic component of the nanocomposites may help improve the interaction between PEI and the PCR components. The developed PEIbased derivatives or nanocomposites may be used as efficient additives to enhance other PCR systems for different biomedical applications.

Keywords: polyethyleneimine, gold nanoparticles, multiwalled carbon nanotubes, nanocomposites, polymerase chain reaction, specificity, efficiency

\section{Introduction}

Developed in 1983 by Mullis, ${ }^{1}$ the polymerase chain reaction (PCR) has been identified as a fundamental technique in contemporary molecular biology and clinical medicine. This gene-amplification technique can increase the number of copies of target genes by six orders of magnitude. ${ }^{2}$ The unique features of PCR enable its wide applications in sequencing, ${ }^{3}$ pathogen detection in food/environment/animals, ${ }^{4-8}$ and genetic analysis. ${ }^{9}$ However, the specificity of PCR does not always match its remarkable sensitivity. ${ }^{10}$ The development of various additives to enhance the specificity and efficiency of PCR is essential. Besides the conventionally used small molecular 
additives, such as formamide, ${ }^{11}$ tetramethylammonium chloride ${ }^{12}$ and its derivatives, ${ }^{13}$ and betaine, ${ }^{14}$ nanoparticles (NPs) have received considerable attention due to their unique physicochemical properties, which are significantly different from those of their bulk counterparts. Various NP systems, including nanogold, ${ }^{15-18}$ carbon nanotubes (CNTs), ${ }^{19}$ carbon nanopowder, ${ }^{20}$ magnetic NPs, ${ }^{21}$ semiconducting NPs,,${ }^{22,23}$ and dendrimers ${ }^{24}$ have been used as additives to improve the PCR specificity and efficiency. Nonspecific amplification problems can be overcome in the presence of NPs.

In our previous studies, ${ }^{24,25}$ we showed that electrostatic interaction between the PCR components and positively charged dendrimers or dendrimer-entrapped gold NPs (Au DENPs) plays a major role in optimizing an error-prone two-round PCR system. Our studies revealed that amineterminated dendrimers have a higher PCR-enhancing effect than those with terminal acetyl and carboxyl groups. Similarly, in the presence of amine-terminated Au DENPs, the PCRenhancing effect is more pronounced than in the scenario of the dendrimers without Au DENPs. ${ }^{25}$ In another study, ${ }^{26}$ we showed that polyethyleneimine (PEI)-modified multiwalled CNTs (MWCNT/PEI) are able to improve the specificity and efficiency of the error-prone two-round PCR system, whereas after acetylation of the PEI amines on the surface of the MWCNTs, the composites are unable to improve PCR specificity and efficiency. All these studies suggest that effective electrostatic interaction between the NPs and PCR components could be the dominating mechanism for enhancing PCR. We also showed ${ }^{26}$ that, as a branched polymer, PEI itself is able to achieve a similar PCR-enhancing effect to that of MWCNT/ PEI. Since the structure of PEI polymer is significantly different from that of NPs (eg, dendrimers, Au DENPs, and MWCNTs), it is logical to explore whether the modification and degree of modification of the PEI amines leads to alteration of the PCR-enhancing effect, which would be helpful for further illustration of the PCR-enhancing mechanism. In addition, since the entrapment of AuNPs within amine dendrimers is able to significantly enhance the PCR due to the reservation of the three-dimensional spherical dendrimer structure, ${ }^{25}$ it is also interesting to investigate the influence of the PEI support (MWCNT for the case of MWCNT/PEI and AuNPs for the case of PEI-stabilized AuNPs) on the PCR enhancing effect of PEI polymer. This is because the electrostatic interaction between PEI polymer and PCR components may be different from that between NP-supported PEI and the PCR components.

In this present study, we explored a new application of branched PEI-based derivatives and hybrid nanocomposites as PCR enhancers. The positively charged amine-terminated pristine PEI was modified with acetic anhydride or succinic anhydride to generate PEI with charge neutral surfaces $\left(\mathrm{PEI}_{-} \mathrm{Ac}_{\mathrm{n}}\right.$ [n denotes the percentage of acetylation]; PEI-PEGAc) or negative surface charge (PEI-SAH). We systematically investigated the effect of the surface charge and surface modification of PEI-based derivatives on the specificity and efficiency of a nonspecific PCR system, which is different from the error-prone two-round PCR system extensively investigated in our previous studies. ${ }^{24-26}$ In addition, the effect of the inorganic support of PEI (in the cases of MWCNT/PEI and PEI-stabilized AuNPs) on the PCRenhancing performance of PEI was also investigated. Possible molecular mechanisms were discussed. To our knowledge, this is the first comprehensive report relating to the optimization of a nonspecific PCR system using PEI with different surface-charge polarities and hybrid nanocomposites.

\section{Methods and materials Materials}

Amine-terminated pristine branched PEI (molecular weight $=25,000$ ), acetic anhydride, and succinct anhydride were purchased from Aldrich (St Louis, MO). The water used in all the experiments was purified using a Milli-Q Plus 185 water purification system (Millipore, Billerica, MA) with a resistivity higher than $18 \mathrm{M} \Omega \mathrm{cm}$. The derivatives of PEI with positively charged (PEI-Ac s0 $^{\text {) }}$, charge-neutral (PEI-Ac, PEI-PEG-Ac), and negatively charged (PEI-SAH) surface groups were synthesized and characterized in our previous work. ${ }^{27}$ The structures of the PEI derivatives are schematically illustrated in Figure 1. MWCNTs and MWCNT/PEI were synthesized in our previous work. ${ }^{28-30}$ Colloid AuNPs with a diameter of $5 \mathrm{~nm}$ and $20 \mathrm{~nm}\left(0.01 \% \mathrm{HAuCl}_{4}\right)$ were purchased from Sigma-Aldrich.

\section{Synthesis and characterization of PEl-stabilized gold nanoparticles}

PEI-stabilized AuNPs (Au/PEI) were synthesized according to the procedures described in the literature, with slight modification. ${ }^{31,32}$ Briefly, PEI $(6 \mathrm{mg})$ was dissolved into $10 \mathrm{~mL}$ water, and then $\mathrm{HAuCl}_{4}(48.6 \mathrm{mg} / \mathrm{mL})$ dissolved in $100 \mu \mathrm{L}$ water was added dropwise to the PEI solution while stirring. The reaction was stopped after 5 days. The obtained $\mathrm{Au} / \mathrm{PEI}$ in aqueous solution without further purification was stored at $4^{\circ} \mathrm{C}$ before use. The synthesized $\mathrm{PEI} / \mathrm{Au}$ was characterized by ultraviolet-visible (UV-vis) spectroscopy and transmission electron microscopy. UV-Vis spectra were collected using a Lambda $25 \mathrm{UV}-\mathrm{Vis}$ spectrometer (PerkinElmer, Waltham, OH). Transmission 


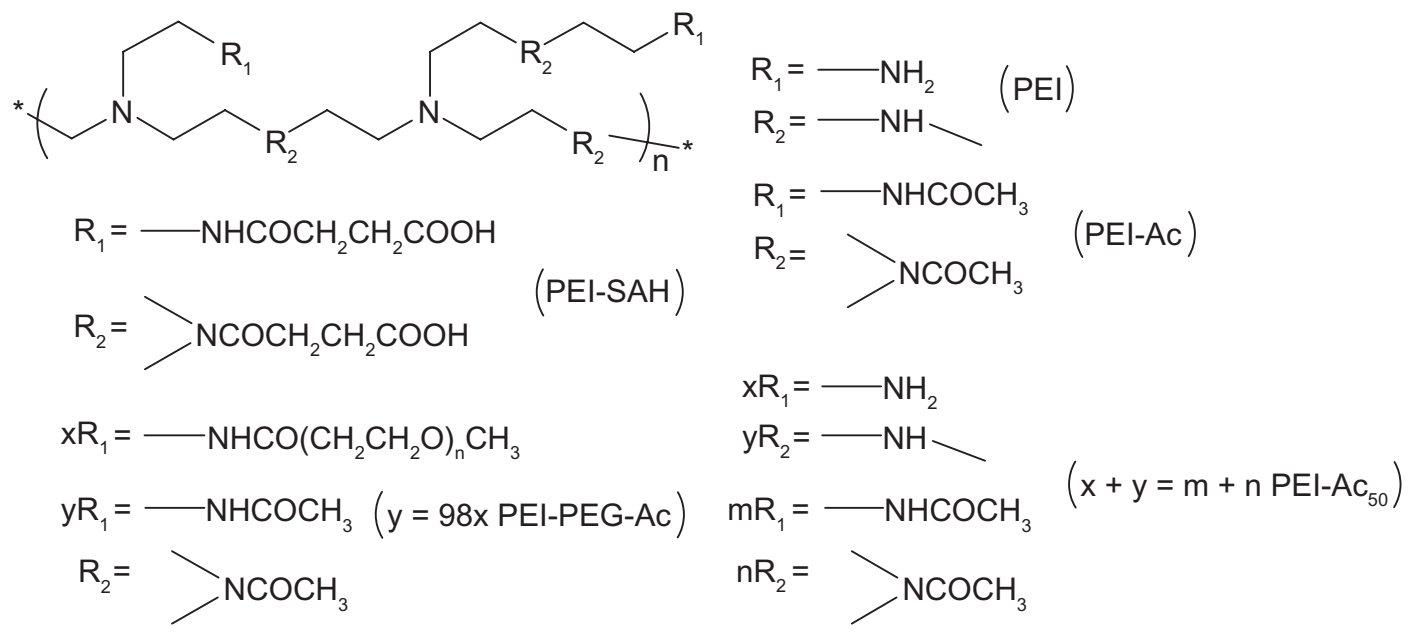

Figure I Schematic illustration of the structures of PEI, PEI-SAH, PEI-Ac, PEI-PEG-Ac, and PEI-Ac $c_{50}$.

Note: $x, y, m$, and $n$ represent the number of $R_{1}$ or $R_{2}$ groups in different $P E I$ derivatives, respectively.

Abbreviations: PEI, polyethyleneimine; PEI-SAH, negatively charged carboxyl-terminated PEI (SAH denotes succinamic acid groups); PEI-Ac, completely acetylated PEI; PEI-PEG-Ac, neutralized PEI modified with both polyethylene glycol and acetyl groups; PEI-Ac ${ }_{50}$, partially (50\%) acetylated PEI.

electron microscopy was performed using a JEOL 2010F analytical electron microscope with an accelerating voltage of $200 \mathrm{kV}$ (JEOL Co, Ltd, Tokyo, Japan). Five microliters of an aqueous solution of sample was dropped onto a carboncoated copper grid and air dried before measurement. The size distribution histogram of the sample was measured using ImageJ software (WS Rasband, National Institutes of Health, Bethesda, MD). Three hundred NPs from different images were randomly selected to analyze the size distribution.

\section{Optimization of a nonspecific PCR system}

In order to test the effect of the additives on PCR, we set up a nonspecific PCR system. In the first-round PCR, a 396 bp target DNA segment was amplified from Pseudomonas aeruginosa genome DNA (extracted in our laboratory) by using one pair of primers with high specificity. Then the PCR products of the first round (diluted 1000 times) were used as the template for the second-round PCR amplification using the same primers. PCR reagents were mixed in a final volume of $25 \mu \mathrm{L}$ according to the following conditions: $10 \mathrm{mM}$ Tris$\mathrm{HCl}$ ( $\mathrm{pH} 8.8$ ), $50 \mathrm{mM} \mathrm{KCl}, 1.5 \mathrm{mM} \mathrm{MgCl}_{2}, 0.2 \mu \mathrm{M}$ primers (Shanghai Sangon Biological Engineering and Technology and Service Co, Ltd, Shanghai, China), $0.25 \mathrm{mM}$ each deoxyribonucleoside triphosphate (Takara Bio Inc), 0.025 U/ $\mu \mathrm{L}$ Taq DNA polymerase (Takara Bio Inc, Shiga, Japan), and $0.8 \mathrm{ng} / \mu \mathrm{L}$ P. aeruginosa genome DNA. The PCR procedure was: 2 minutes at $94^{\circ} \mathrm{C}$ for pre-denaturation, followed by 27 cycles of: 30 seconds at $94^{\circ} \mathrm{C}, 30$ seconds at $60^{\circ} \mathrm{C}$, and 30 seconds at $72^{\circ} \mathrm{C}$. Then the cycling was terminated after
5 minutes' incubation at $72^{\circ} \mathrm{C}$. The sequences of the primers were as follows: primer 1: 5'-GACAACGCCCTCAGCATCACCAGC-3', and primer 2: 5'-CGCTGGCCCATTCGCTCCAGCGCT-3'. Amplifications were carried out in an S1000 ${ }^{\mathrm{TM}}$ Thermal Cycler (Bio-Rad Inc, Hercules, CA).

PCR products $(3 \mu \mathrm{L})$ were examined by using $1.5 \%$ agarose gel electrophoresis with $0.6 \mu \mathrm{L}$ loading buffer. All the products of PCR were sequenced in order to determine the fidelity of PCR. The effectiveness of the PEI derivatives and hybrid nanocomposites was described through the assignment of two densitometric quantifications, termed specificity and efficiency. From electropherograms, the specificity of amplification was calculated as a ratio of the densitometric value of the specific band and that of all bands amplified by PCR, including undesired nonspecific bands. By definition, maximal value of specificity, in the absence of nonspecific bands, equals 1.0. The efficiency of an additive is defined as a ratio of the densitometric value of the target DNA band determined after PCR to 500 bp of DL2000 DNA marker, which is assigned to a value of 1.0. The concentration of each additive that enabled the PCR to produce the most specific and the brightest target band (maximum efficiency) on the gel was identified to be the optimum concentration.

\section{Results and discussion}

The effect of PEl-based derivatives on the nonspecific PCR system

In the designed nonspecific PCR system, the specific band was able to be observed in the first round. However, even if the same primers were used, nonspecific bands in the 
second-round PCR always appeared. Below the DNA segment with a target band of $396 \mathrm{bp}$, the nonspecific band is visible (Figure 2, Lane 1 for each panel), which may be caused by side-reaction products. ${ }^{20}$ Therefore, as in our previous studies utilizing an error-prone two-round PCR system, ${ }^{24-26}$ this PCR system was selected as a model to test the PCR-enhancing effect using PEI-based derivatives.
The PEI-based derivatives with different surface functionalities were separately added into the PCR reaction mixture at different concentrations to optimize the PCR, and the PCR products were analyzed by agarose gel electrophoresis (Figure 2). Five different PEI derivatives were tested: pristine amine-terminated PEI; 50\% acetylated PEI (PEI-Ac $\left.{ }_{50}\right) ; 100 \%$ acetylated PEI (PEI-Ac); negatively
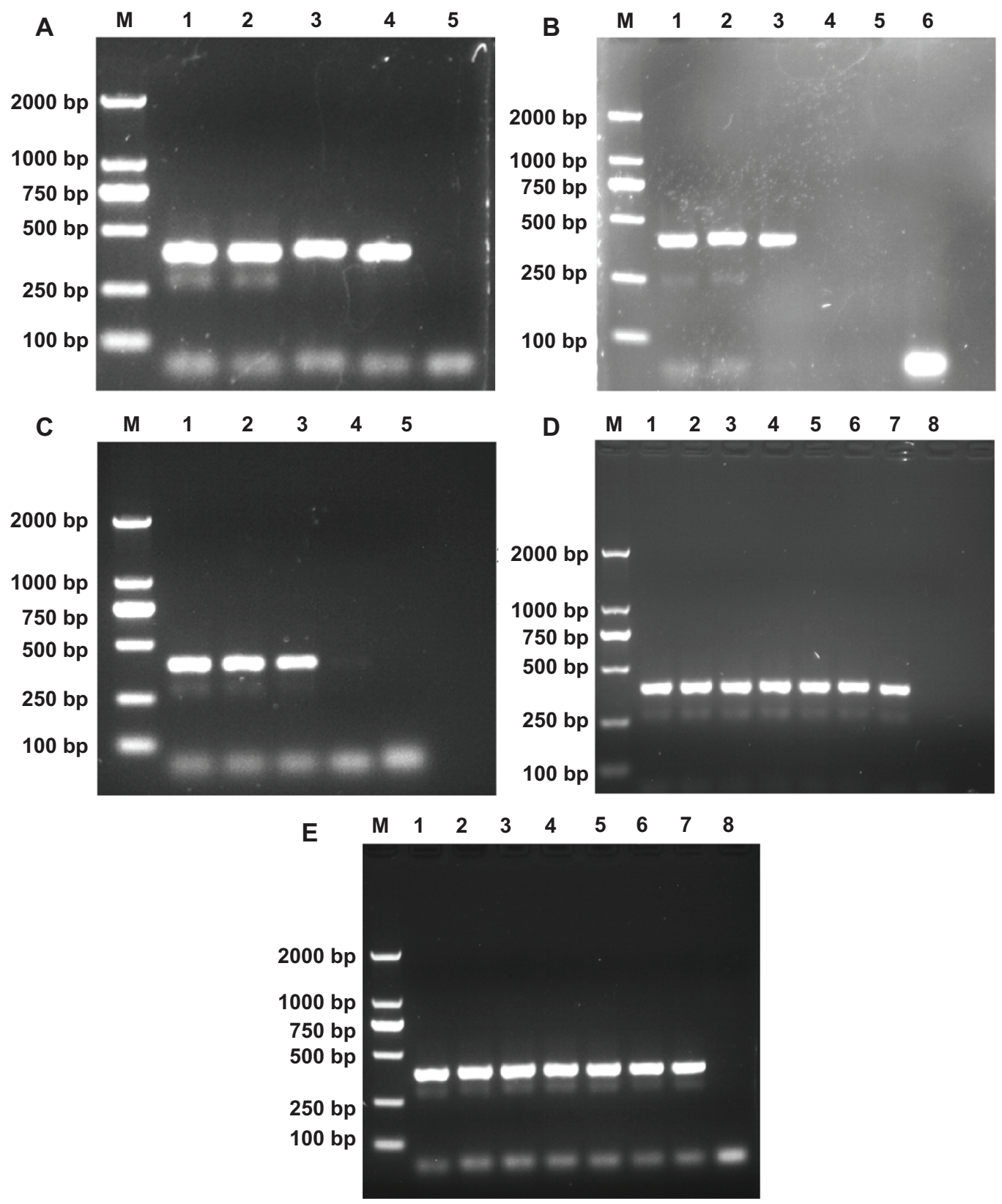

Figure 2 The effects of PEl-based derivatives on the nonspecific polymerase chain reaction (PCR) system. Lane M is the DL 2000 marker, and the rightmost lane in each panel is the negative control amplified without template and additives. (A) PEl was added into the PCR mixture. From lane I to 4, its final concentration is $0,20,40,60 \mu g / L$, respectively. (B) PEI-Ac ${ }_{50}$ was added into the PCR mixture. From lane I to line 5, its final concentration is 0, 45, 90, II 2, and I35 $\mu$ g/L, respectively. (C) PEI-Ac was added into the PCR mixture. From lane I to 4 , its final concentration is $0,36,360$, and $720 \mu \mathrm{g} / \mathrm{L}$, respectively. (D) PEI-SAH was added into the PCR mixture. From lane I to 7 , its final concentration is $0,17,34,102,170,238$, and $340 \mathrm{mg} / \mathrm{L}$, respectively. (E) PEI-PEG-Ac was added into the PCR mixture. From lane I to 7 , its final concentration is 0 , I6.4, 32.8, 65.6, 84, I3I, and $164 \mathrm{mg} / \mathrm{L}$, respectively.

Abbreviations: PEl, polyethyleneimine; PEI-Ac ${ }_{50}$, partially $(50 \%)$ acetylated PEI; PEI-Ac, completely acetylated PEl; PEI-SAH, negatively charged carboxyl-terminated PEI (SAH denotes succinamic acid groups); PEI-PEG-Ac, neutralized PEI modified with both polyethylene glycol and acetyl groups. 
charged PEI (PEI-SAH), and PEI modified with both PEG and acetamide (PEI-PEG-Ac). It is clear that PEI, PEI-Ac ${ }_{50}$, and PEI-Ac improve the efficiency and specificity of the nonspecific PCR system. The semiquantitative results of the PCR specificity and efficiency data in the presence of each additive are listed in Table 1. When PEI, PEI-Ac ${ }_{50}$, and PEIAc were respectively added into the PCR mixture, the nonspecific band started to decrease as the additive concentration increased and vanished at the optimal concentration. When the concentrations of the additives exceeded their optimum concentrations, the amplification of target band and the nonspecific products were significantly inhibited, in agreement with literature data. ${ }^{15,22,24-26}$ The detailed mechanism remains unclear and it has been difficult to explain why highly concentrated PCR additives are able to inhibit the PCR. The optimum concentrations of PEI, PEI-Ac ${ }_{50}$, and PEI-Ac were estimated to be 40,90 , and $360 \mu \mathrm{g} / \mathrm{L}$, respectively, which can be transformed to PEI concentrations of 40, 66, and $209 \mu \mathrm{g} / \mathrm{L}$, respectively ${ }^{27}$ with an order of PEI $<$ PEI-Ac ${ }_{50}<$ PEI-Ac. This indicates that the PCR-enhancing effect of the PEI derivatives follows the order of PEI $>$ PEI-Ac $\mathrm{c}_{50}>$ PEI-Ac. Our previous work has shown that electrostatic interaction between dendrimers or MWCNTs and the PCR components may play an important role in enhancing PCR specificity and efficiency. ${ }^{24,26}$ The used DNA template, DNA primers, and the DNA polymerase (Taq DNA polymerase) with an isoelectric point of $6.03^{33}$ are all negatively charged under the PCR conditions ( $\mathrm{pH}$ 7.4), enabling effective electrostatic interaction with the positively charged PCR additives. As the degree of acetylation increases, the optimum concentration of PEI tends to increase, which is presumably due to the decreased number of surface amine groups, making the electrostatic interaction between PEI derivatives and PCR components weaker. These results are consistent with those reported in our previous study related to dendrimerbased additives with different degrees of acetylation. ${ }^{24} \mathrm{It}$ is interesting to note that even for PEI-Ac, the surface amines of PEI cannot be completely acetylated due to the formation of ion pairs between the formed acetic acid and the PEI amines during the acetylation reaction. ${ }^{34}$ Therefore, weak interaction between the PEI-Ac and PCR components still contributes to the PCR-enhancing effect. In contrast, the negatively charged PEI-SAH and the neutral PEI-PEG-Ac were unable to enhance the efficiency and specificity of PCR even at the concentration of three orders of magnitude higher than that of PEI, which is possibly due to the lack of interaction between the negatively charged PCR components and the negatively charged PEI-SAH or neutralized PEI-PEG-Ac with antifouling properties. ${ }^{35}$ These results imply that the surface interaction between additives and PCR components plays an important role in enhancing the specificity and efficiency of PCR.

\section{The effect of PEl-based hybrid nanocomposites on the nonspecific PCR system}

Our earlier study shows that PEI can be modified onto the surface of MWCNTs ${ }^{28}$ and both PEI and MWCNT/PEI are able to improve the specificity and efficiency of an errorprone two-round PCR system. ${ }^{26}$ Since the PCR-enhancing effect of the PEI-based derivatives is primarily based on the electrostatic interaction of the polymers and the PCR components, it would be interesting to compare PEI and MWCNT/PEI in terms of their enhancement of the nonspecific PCR system in this study, which is very different from the PCR system reported in our previous studies. ${ }^{24-26}$ With the supporting MWCNTs, the electrostatic interaction between PEI and the PCR components may be enhanced due to the enlarged surface area and increased binding sites, therefore the PCR-enhancing effect may be different. In this study, the PCR-enhancing effect of pristine acid-treated MWCNTs, MWCNT/PEI, and PEI was compared.

Table I Efficiency, specificity, and optimum concentrations of additives in a nonspecific polymerase chain reaction system

\begin{tabular}{lllll}
\hline Additive & $\begin{array}{l}\text { Optimum } \\
\text { concentration }(\mu \mathrm{g} / \mathrm{L})\end{array}$ & $\begin{array}{l}\text { Optimum PEI } \\
\text { concentration }(\mu \mathrm{g} / \mathrm{L})\end{array}$ & Maximal efficiency & Maximal specificity \\
\hline MWCNTs & 6200 & - & 1.60 & 1.00 \\
MWCNT/PEI (PEI 30.6\%) & & 29 & 1.25 & 1.00 \\
PEI & 94 & 40 & 1.48 & 1.00 \\
PEI-Ac ${ }_{50}($ PEI 73.5\%) & 90 & 66 & 1.44 & 0.97 \\
PEI-Ac (PEI 58.2\%) & 90 & 209 & 1.05 & 1.00 \\
Au/PEI (PEI 68\%) & 360 & 36 & 1.08 & 0.96 \\
\hline
\end{tabular}

Notes: ${ }^{\text {TT }}$ The percentage of PEl in the hybrid nanocomposites can be found in Shen et al; ${ }^{28}$ bthe percentage of PEI was calculated according to Wen et al, ${ }^{27}$ cthe percentage of $\mathrm{PEI}$ was calculated based on the weight ratio of PEI and $\mathrm{HAuCl} 4$ in the reaction (see Methods and materials).

Abbreviations: PEl, polyethyleneimine; MWCNTs, multiwalled carbon nanotubes; MWCNT/PEI, PEI-modified MWCNTs; PEI-Ac50, partially (50\%) acetylated PEI; PEI-Ac, completely acetylated PEI; Au/PEI, PEI-stabilized gold nanoparticles. 

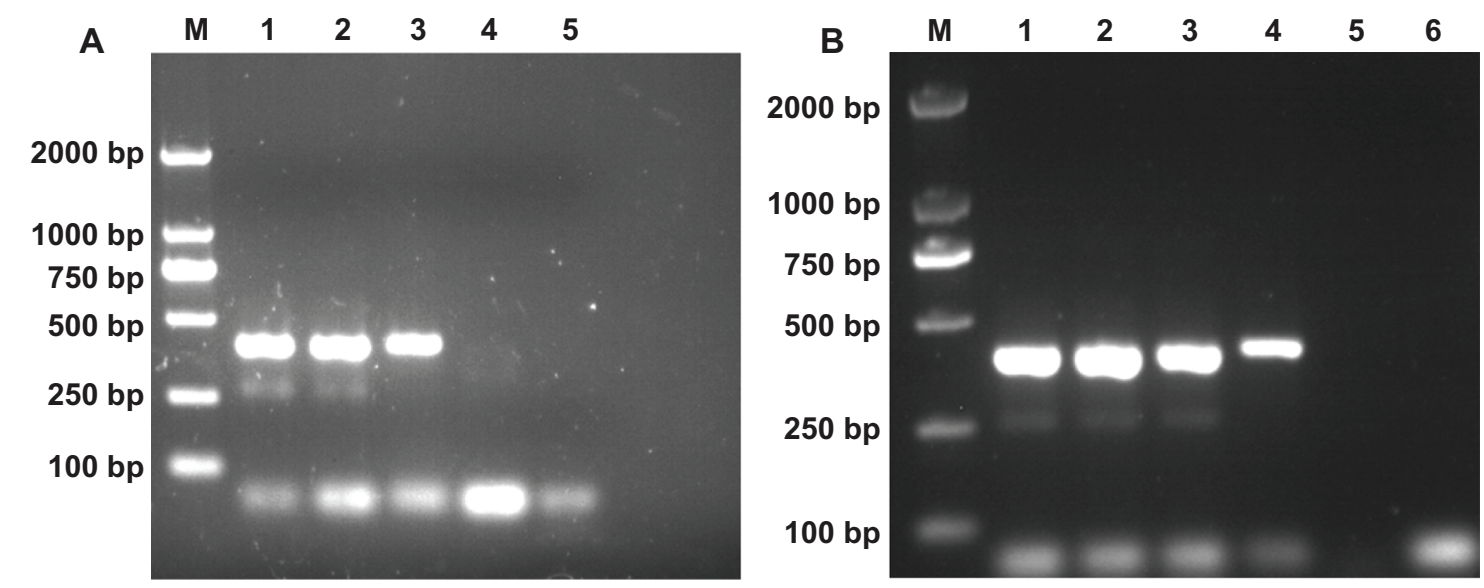

Figure 3 The effects of MWCNTs and MWCNT/PEI on the specificity of the nonspecific polymerase chain reaction system. Lane M is the DL 2000 marker, and the rightmost lane is negative control in each panel. (A) MWCNT was added into the polymerase chain reaction mixture. From lane I to 4, its final concentration is 0 , 3.I, 6.2, and $12.4 \mathrm{mg} / \mathrm{L}$, respectively. (B) MWCNT-PEl was added into the polymerase chain reaction mixture. From lane I to 5 , its final concentration is $0,23.6,47.2,94.2$, and $188.8 \mu \mathrm{g} / \mathrm{L}$, respectively.

Abbreviations: MWCNTs, multiwalled carbon nanotubes; MWCNT/PEl, polyethyleneimine-modified multiwalled carbon nanotubes.

Figure 3 shows the results of PCR optimized using the tested additives. With the addition of the MWCNTs and MWCNT/PEI, the original nonspecific product disappears, indicating that MWCNTs and MWCNT/PEI are all able to improve the specificity and efficiency of the nonspecific PCR system. The optimum concentrations of the MWCNTs, MWCNT/PEI, and PEI were estimated to be $6200 \mu \mathrm{g} / \mathrm{L}$, $94 \mu \mathrm{g} / \mathrm{L}$, and $40 \mu \mathrm{g} / \mathrm{L}$, respectively (Table 1, Figure $2 \mathrm{~A}$ ). It is clear that the modification of MWCNTs with PEI makes the required optimum concentration of PEI $(29 \mu \mathrm{g} / \mathrm{L})$ much lower than that of free PEI $(40 \mu \mathrm{g} / \mathrm{L})$, indicative of the strong interaction between the negatively charged PCR components and the positively charged MWCNT/PEI. MWCNTs without PEI modification are able to improve the PCR specificity and efficiency; however, the required concentration is two orders of magnitude higher than that of MWCNT/PEI.

With the support of MWCNTs, the interaction of PCR components with PEI could be significantly enhanced due to the increased binding sites, making the PCR-enhancing effect much more effective. To further prove this mechanism, we prepared Au/PEI, where the PEI molecules are supported onto the surface of AuNPs according to the procedures described in the literature. ${ }^{31,32}$ The dual roles of reducing agent and stabilizer played by PEI enabled the successful formation of PEI-stabilized AuNPs. After a 5-day reaction, the solution became wine red (Figure 4, inset), indicating the formation of AuNPs. UV-Vis spectroscopy study reveals that the synthesized Au/PEI displays a surface plasmon band at $532 \mathrm{~nm}$, which is typical for AuNPs (Figure 4). The formed $\mathrm{Au} / \mathrm{PEI}$ displays a spherical shape and has a mean diameter of $7.6 \mathrm{~nm}$ with a narrow size distribution (Figure 5). The somewhat aggregated particles shown in Figure 5A are presumably due to the transmission electron microscopy sample preparation method. Air-drying of the aqueous suspension of the samples before transmission electron microscopy measurement may lead to a partial aggregation or interconnection of particles, in agreement with previous literature. ${ }^{36}$

The addition of $\mathrm{Au} / \mathrm{PEI}$ was able to enhance the nonspecific PCR, similar to the PEI polymer (Figure 6). The optimum concentration of PEI in Au/PEI nanocomposite was measured to be $36 \mu \mathrm{g} / \mathrm{L}$, which is lower than that of free PEI $(40 \mu \mathrm{g} / \mathrm{L})$. This further suggests that the support of PEI by AuNPs with a

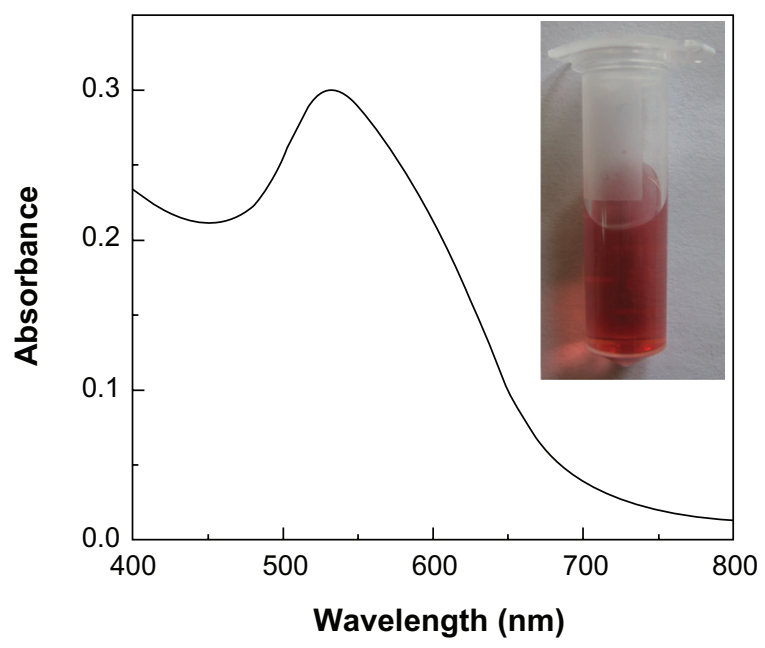

Figure 4 Ultraviolet-visible spectrum of the polyethyleneimine-stabilized gold nanoparticles (Au/PEl). The inset shows a photograph of the nanoparticles suspended in water. 

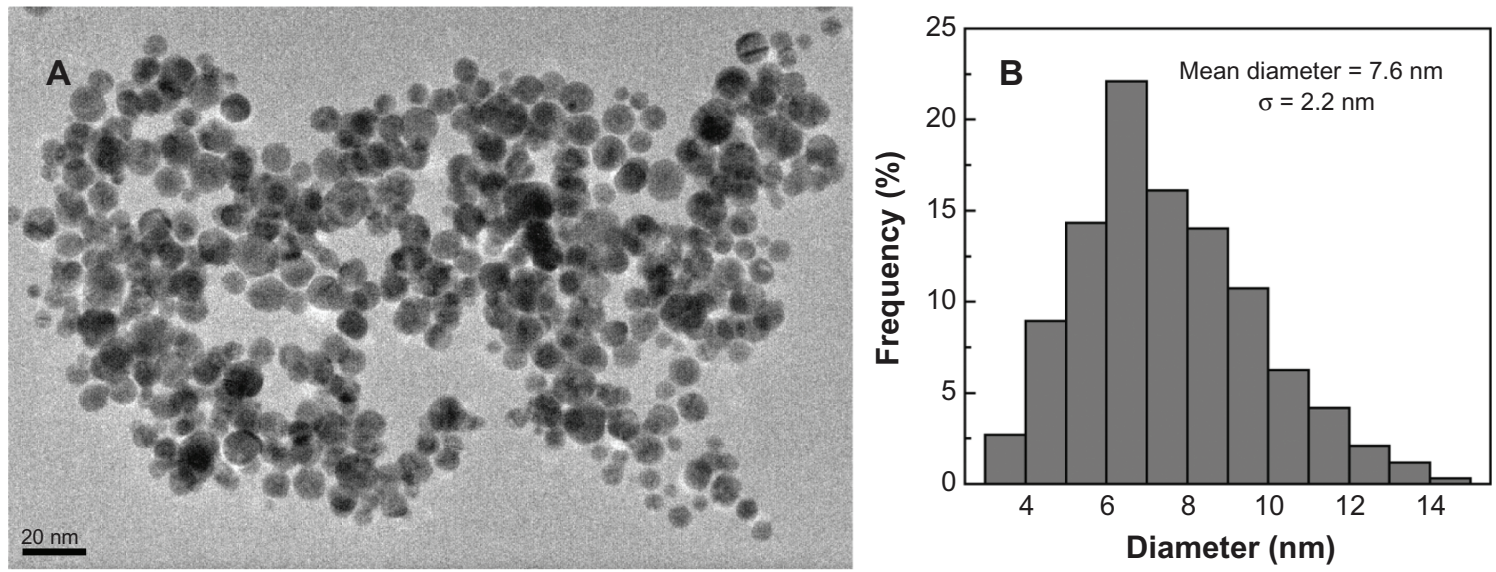

Figure 5 Transmission electron microscopy image (A) and size distribution histogram (B) of the polyethyleneimine-stabilized gold nanoparticles (Au/PEI).

mean diameter of $7.6 \mathrm{~nm}$ could also improve the electrostatic interaction between PEI and the PCR components, thereby enhancing the PCR more efficiently than free PEI. This result is consistent with that obtained using MWCNT/PEI as an additive. It should be noted that the approach to synthesizing $\mathrm{Au} / \mathrm{PEI}$ is quite "green" (ie, not harmful to the environment since there is no need to use additional toxic chemicals and no byproducts can be formed in the reaction that need additional purification steps) - without any additional chemical reducing agents. Only a quite small amount of $\mathrm{HCl}$ is expected to be produced after the reaction. The formed $\mathrm{HCl}$ is likely to form ion pairs with the PEI surface amines, which makes their purification by washing with water (centrifugation or dialysis) difficult. Given the fact that the reaction solution to form $\mathrm{Au} / \mathrm{PEI}$ is quite dilute and that the used $\mathrm{Au} / \mathrm{PEI}$ in

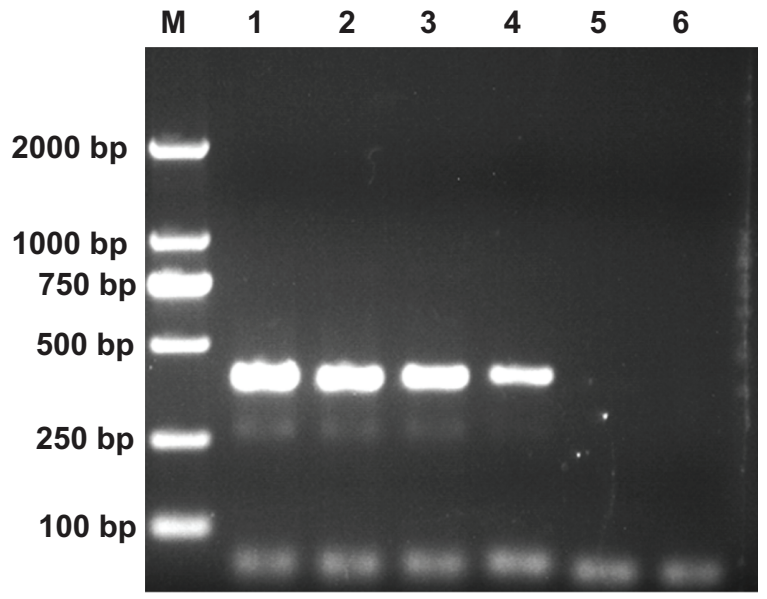

Figure 6 The effect of the polyethyleneimine-stabilized gold nanoparticles on the nonspecific polymerase chain reaction system. Lane M is the DL 2000 marker, and the rightmost lane is the negative control. From lane I to 5 , the final polyethyleneimine concentration is $0,12.5,25,36$, and $75 \mu \mathrm{g} / \mathrm{L}$, respectively. the PCR mixture solution (pH 8.8 buffer) is also quite dilute with a concentration less than ppm level, the $\mathrm{HCl}$ that forms ion pairs with PEI surface amines is likely to be dissociated in the PCR mixture solution and would not have any appreciable impact on the PCR amplification. Additionally, commercially available colloid AuNPs with a diameter of 5 or $20 \mathrm{~nm}$ were also used to enhance the nonspecific PCR in comparison with the Au/PEI. Our results showed that colloid AuNPs of two different sizes were able to enhance the PCR (Figure S1, Supporting information), however the optimal Au concentrations for $5 \mathrm{~nm}$ AuNPs and $20 \mathrm{~nm}$ AuNPs were one and two orders of magnitude higher than that of the $\mathrm{Au} /$ PEI, respectively (Table 2), implying that the coating of positively charged PEI onto the surface of AuNPs for the $\mathrm{Au} / \mathrm{PEI}$ is very important to allow for effective electrostatic interaction with the negatively charged PCR components, thereby greatly enhancing the PCR.

\section{The amplification fidelity of nonspecific PCR using PEI derivatives}

An ideal PCR additive should not interfere with the final PCR products. Therefore, it is important to know whether the

Table 2 Efficiency, specificity, and optimum gold ( $A u)$ concentrations of additives in a nonspecific polymerase chain reaction system

\begin{tabular}{llll}
\hline Additive & $\begin{array}{l}\text { Optimum Au } \\
\text { concentration }(\mu \mathrm{g} / \mathrm{L})\end{array}$ & $\begin{array}{l}\text { Maximal } \\
\text { efficiency }\end{array}$ & $\begin{array}{l}\text { Maximal } \\
\text { specificity }\end{array}$ \\
\hline $\mathrm{Au} / \mathrm{PEI}(\mathrm{Au} \mathrm{32 \%})^{\mathrm{a}}$ & 17 & 1.08 & 0.96 \\
$\mathrm{Au}(5 \mathrm{~nm})^{\mathrm{b}}$ & 232 & 1.10 & 1.00 \\
$\mathrm{Au}(20 \mathrm{~nm})^{\mathrm{b}}$ & 3480 & 1.00 & 1.00 \\
\hline
\end{tabular}

Notes: ${ }^{\text {TT }}$ The percentage of Au was calculated based on the weight ratio of PEI and $\mathrm{HAuCl} 4$ in the reaction (see Methods and materials); bthe Au concentration was calculated based on the manufacturer's specification.

Abbreviation: Au/PEl, polyethyleneimine-stabilized gold nanoparticles. 
CLUSTAL X(1.83) multiple sequence alignment

1p 1 TCGCTCCAGCGCTTTTCCCGGCGCGGCTGGGCCTGGGCCATGACCACGCTGACC 54 2p 1 CGATCGCTGGCCCCATTCGCTCCAGCGCTTTTCCCGGCGCGGCTGGGCCTGGGCCATGACCACGCTGACC 70

1p 55 CCGGCATGGCTGATGGCGAGCGTCGGCTGCATCTCGTTGCTCTCGTGCGCCCTGACGAAGAAGGTGGCAT 124 2p 71 CCGGCATGGCTGATGGCGAGCGTCGGCTGCATCTCGTTGCTCTCGTGCGCCCTGACGAAGAAGGTGGCAT 140

1p 125 CGCGCGCCAGCTTCGCCAGCAACTCGTCGCCCATCTCGATGGTGTAGATCGGCGACATGTGGCTGAGCTG 194 2p 141 CGCGCGCCAGCTTCGCCAGCAACTCGTCGCCCATCTCGATGGTGTAGATCGGCGACATGTGGCTGAGCTG 210

1p 195 GTTACCGGCGTTCAGTTCGTGGATGAACACCTTGATGTTCGAAGGCTTCTCGTGGCCGATCGGCACCAGC 264 2p 211 GTTACCGGCGTTCAGTTCGTGGATGAACACCTTGATGTTCGAAGGCTTCTCGTGGCCGATCGGCACCAGC 280

1p 265 CAGTTCAGCGACCAACTGCCGCGCGCCTGGCGCGTGTAGCTGTAGCGCACCGGCTTGTTCGGCTCGACGC 334 2p 281 CAGTTCAGCGACCAACTGCCGCGCGCCTGGCGCGTGTAGCTGTAGCGCACCGGCTTGTTCGGCTCGACGC 350

1p 335 CGCCTTCGAGGCGGATGGTCAGGCCGTCGCTGGTGATGCTGAGGG--CGTTGTCA 387

2p 351 CGCCTTCGAGGCGGATGGTCAGGCCGTCGCTGGTGATGCTGAGGGGCGTTGTC 403

Figure 7 Sequencing results to test the amplification fidelity of polyethyleneimine-assisted polymerase chain reaction. I p: without polyethyleneimine; $2 \mathrm{p}$ : with polyethyleneimine. CLUSTAL X(1.83) was used for DNA sequence alignment in a Windows XP computer system.

studied PCR additives influence the fidelity of target DNA product. All the PCR products in the absence or presence of the different additives were sequenced to confirm the fidelity of the PCR. For example, we used the primer 1, primer 2, and 396 bp PCR product in lane 3 of Figure 2A (in the presence of PEI as additive) for sequencing tests. The sequencing result is shown in Figure 7. It is clear that the sequence of the PCR product in the presence of $\mathrm{PEI}$ additive is quite consistent with that in the absence of PEI. All our sequencing data confirmed that the PCR in the presence of the effective additives of PEI derivatives and hybrid nanocomposites had similar fidelity to that of the PCR in the absence of the additives.

\section{Conclusion}

In summary, PEI-based derivatives and hybrid nanocomposites were utilized as additives to enhance the specificity and efficiency of a nonspecific PCR system. Our data show that the positively charged PEI derivatives are able to enhance the specificity and efficiency of the nonspecific PCR system, whereas negatively charged PEI derivatives and neutralized PEI-PEG-Ac with antifouling property have no such capacity, even at concentrations three orders of magnitude higher than that of PEI. In addition, the PEI polymer supported onto the surface of MWCNTs or AuNPs appears to have a more pronounced effect on the enhancement of the PCR. Our results clearly suggest that the electrostatic interaction between PEI derivatives with PCR components plays an important role in enhancing PCR specificity and efficiency. PEI and PEI-based hybrid nanocomposites may be used as effective additives for enhancing other PCR systems for different biomedical applications.

\section{Acknowledgments}

This research is financially supported by the Program for New Century Excellent Talents in University, the National Natural Science Foundation of China (20974019, 81101150, and 81071747), the Fund of the Science and Technology Commission of Shanghai Municipality (11 nm0506400), the Fundamental Research Funds for the Central Universities (for XC, RG, MS, and XS), and the Key Laboratory of Textile Science and Technology, Ministry of Education, "111 Project", B07024. JW thanks the Shanghai Education Committee Key Discipline and Specialties Foundation (J50208) and Shanghai Pujiang Program (10PJ1406400). XS gratefully acknowledges the Fundação para a Ciência e a Tecnologia (FCT) and Santander Bank for the Invited Chair in Nanotechnology.

\section{Disclosure}

The authors report no conflicts of interest in this work.

\section{References}

1. Bartlett J, Stirling D. A short history of the polymerase chain reaction. Methods Mol Biol. 2003;226(1):3-6.

2. Dieffenbach CW, Dveksler GS. PCR Primer: A Laboratory Manual. New York: Cold Spring Harbor Laboratory Press; 2003.

3. Li H, Rothberg LJ. Label-free colorimetric detection of specific sequences in genomic DNA amplified by the polymerase chain reaction. JAm Chem Soc. 2004;126(35):10958-10961.

4. Lo CC, Chen SC, Yang JZ. Use of real-time polymerase chain reaction (PCR) and transformation assay to monitor the persistence and bioavailability of transgenic genes released from genetically modified papaya expressing nptII and PRSV genes in the soil. J Agr Food Chem. 2007;55(18):7534-7540.

5. Hubalkova Z, Kralik P, Kasalova J, Rencova E. Identification of gadoid species in fish meat by polymerase chain reaction (PCR) on genomic DNA. J Agr Food Chem. 2008;56(10):3454-3459. 
6. Ram S, Vajpayee P, Shanker R. Rapid culture-independent quantitative detection of enterotoxigenic Escherichia coli in surface waters by realtime PCR with molecular beacon. Environ Sci Technol. 2008;42(12): 4577-4582.

7. Koh CG, Tan W, Zhao M, Ricco AJ, Fan ZH. Integrating polymerase chain reaction, valving, and electrophoresis in a plastic device for bacterial detection. Anal Chem. 2003;75(17):4591-4598.

8. Brakstad OG, Aasbakk K, Maeland JA. Detection of Staphylococcus aureus by polymerase chain reaction amplification of the nuc gene. J Clin Microbiol. 1992;30(7):1654-1660.

9. Myakishev MV, Khripin Y, Hu S, Hamer DH. High-throughput SNP genotyping by allele-specific PCR with universal energy-transferlabeled primers. Genome Res. 2001;11(1):163-169.

10. Chou Q, Russell M, Birch DE, Raymond J, Bloch W. Prevention of prePCR mis-priming and primer dimerization improves low-copy-number amplifications. Nucleic Acids Res. 1992;20(7):1717-1723.

11. Sarkar G, Kapelner S, Sommer SS. Formamide can dramatically improve the specificity of PCR. Nucleic Acids Res. 1990;18(24):7465.

12. Chevet E, Lema tre G, Katinka MD. Low concentrations of tetramethylammonium chloride increase yield and specificity of PCR. Nucleic Acids Res. 1995;23(16):3343-3344.

13. Kovarova M, Draber P. New specificity and yield enhancer of polymerase chain reactions. Nucleic Acids Res. 2000;28(13):E70.

14. Rees WA, Yager TD, Korte J, Von Hippel PH. Betaine can eliminate the base pair composition dependence of DNA melting. Biochemistry. 1993;32(1):137-144.

15. Li HK, Huang JH, Lv J, et al. Nanoparticle PCR: Nanogold-assisted PCR with enhanced specificity. Angew Chem Int Ed Engl. 2005;44(32): 5100-5103.

16. Li M, Lin YC, Wu CC, Liu HS. Enhancing the efficiency of a PCR using gold nanoparticles. Nucleic Acids Res. 2005;33(21):e184.

17. Mi L, Wen Y, Pan D, Wang Y, Fan C, Hu J. Modulation of DNA polymerases with gold nanoparticles and their applications in hot-start PCR. Small. 2009;5:2597-2600.

18. Yang W, Mi L, Cao X, Zhang X, Fan C, Hu J. Evaluation of gold nanoparticles as the additive in real-time polymerase chain reaction with SYBR Green I dye. Nanotechnology. 2008;19:255101.

19. Zhang ZZ, Shen CC, Wang MC, Han H, Cao XH. Aqueous suspension of carbon nanotubes enhances the specificity of long PCR. Biotechniques. 2008;44(4):537-544.

20. Zhang ZZ, Wang MC, An HJ. An aqueous suspension of carbon nanopowder enhances the efficiency of a polymerase chain reaction. Nanotechnology. 2007;18(35):355706-355711.

21. Shen HB, Hu M, Wang YB, Zhou HQ. Polymerase chain reaction of nanoparticle-bound primers. Biophys Chem. 2005;115:63-66.

22. Wang L, Zhu Y, Jiang Y, et al. Effects of quantum dots in polymerase chain reaction. J Phys Chem B. 2009;113:7637-7641.

23. Abdul Khaliq R, Sonawane PJ, Sasi BK, et al. Enhancement in the efficiency of polymerase chain reaction by $\mathrm{TiO} 2$ nanoparticles: crucial role of enhanced thermal conductivity. Nanotechnology. 2010;21: 255704.
24. Cao XY, Shi XY, Yang WC, Zhang XD, Fan CH, Hu J. Enhanced specificity and efficiency of polymerase chain reactions using poly(amidoamine) dendrimers and derivatives. Analyst. 2009;134(1): 87-92

25. Chen J, Cao X, Guo R, et al. A highly effective polymerase chain reaction enhancer based on dendrimer-entrapped gold nanoparticles. Analyst. 2012;137:223-228.

26. Cao X, Chen J, Wen S, Peng C, Shen M, Shi X. Effect of surface charge of polyethyleneimine-modified multiwalled carbon nanotubes on the improvement of polymerase chain reaction. Nanoscale. 2011;3: 1741-1747.

27. Wen SH, Zheng FY, Shi XY. Improved biocompatibility of polyethyleneimine modified with acetamide, hydroxyl, carboxyl groups and PEG chains. Proceedings of 2011 International Forum on Biomedical Textile Materials. 2011:189-193.

28. Shen M, Wang SH, Shi X, et al. Polyethyleneimine-mediated functionalization of multiwalled carbon nanotubes: synthesis, characterization, and in vitro toxicity assay. J Phys Chem C. 2009;113(8): 3150-3156.

29. Petersen EJ, Huang Q, Weber WJ Jr. Ecological uptake and depuration of carbon nanotubes by Lumbriculus variegatus. Environ Health Perspect. 2008;116(4):496-500.

30. Petersen EJ, Huang Q, Weber WJ Jr. Bioaccumulation of radiolabeled carbon nanotubes by Eisenia foetida. Environ Sci Technol. 2008;42(8):3090-3095.

31. Mikami T, Takayasu Y, Watanabe J, Hirasawa I. Influence of polyethyleneimine addition on crystal size distribution of Au nanocrystals. Chem Eng Technol. 2011;34:583-586.

32. Sun X, Dong S, Wang E. One-step preparation of highly concentrated well-stable gold colloids by direct mix of polyelectrolyte and $\mathrm{HAuCl} 4$ aqueous solutions at room temperature. J Colloid Interface Sci. 2005;288:301-303.

33. ExPASy Bioinformatics Resource Portal. DNA polymerase I, thermostable. Geneva, Lausanne, Basel, Bern, Zurich: Swiss Institute of Bioinformatics; nd. Available from: http://web.expasy.org/cgi-bin/ compute_pi/pi_tool1?P19821@1-832@average. Accessed February 17. 2011.

34. Majoros IJ, Keszler B, Woehler S, Bull T, Baker JR Jr. Acetylation of poly(amidoamine) dendrimers. Macromolecules. 2003;36(15): 5526-5529.

35. Kim D, Park S, Lee JH, Jeong YY, Jon S. Antibiofouling polymer-coated gold nanoparticles as a contrast agent for in vivo X-ray computed tomography imaging. J Am Chem Soc. 2007;129(24):7661-7665.

36. Shi X, Briseno AL, Sanedrin RJ, Zhou F. Formation of uniform polyaniline thin shells and hollow capsules using polyelectrolyte-coated microspheres as templates. Macromolecules. 2003;36:4093-4098. 


\section{Supplementary figure}
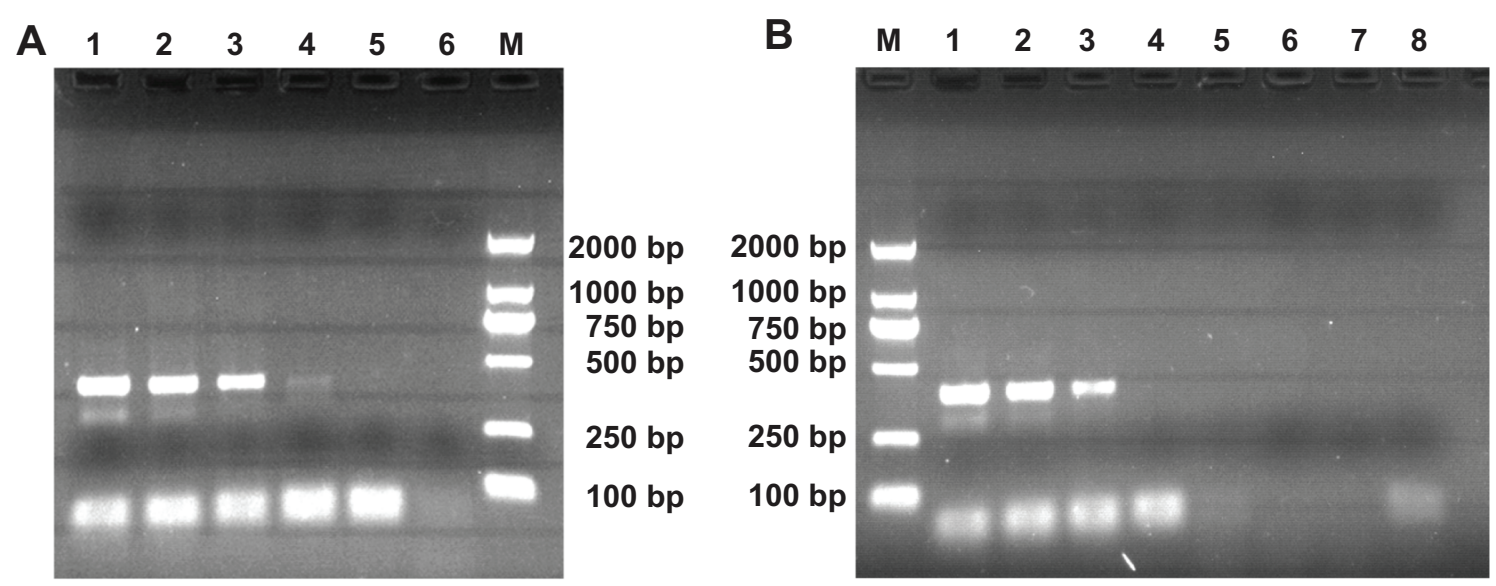

Figure SI The effects of commercial colloid gold nanoparticles (AuNPs) on the nonspecific polymerase chain reaction system. (A) The effect of AuNPs with a diameter of $5 \mathrm{~nm}$. Lane M is the DL 2000 marker, and lane 6 is the negative control amplified without template and additives. AuNPs (5 nm) were added into the polymerase chain reaction mixture. From lane I to 5, its final Au concentration is 0,1 I 6, 232, 348, and $464 \mu \mathrm{g} / \mathrm{L}$, respectively. (B) The effect of AuNPs with a diameter of $20 \mathrm{~nm}$. Lane M is the $\mathrm{DL} 2000$ marker, and lane 8 is the negative control amplified without template and additives. AuNPs (20 nm) were added into the polymerase chain reaction mixture. From lane I to 7 , its final Au concentration is $0,2.32,3.48,6.96,1 \mathrm{I} .6,16.24$, and $23.2 \mathrm{mg} / \mathrm{L}$, respectively.

\section{Publish your work in this journal}

The International Journal of Nanomedicine is an international, peerreviewed journal focusing on the application of nanotechnology in diagnostics, therapeutics, and drug delivery systems throughout the biomedical field. This journal is indexed on PubMed Central, MedLine, CAS, SciSearch $\AA$, Current Contents ${ }^{\circledR} /$ Clinical Medicine,
Journal Citation Reports/Science Edition, EMBase, Scopus and the Elsevier Bibliographic databases. The manuscript management system is completely online and includes a very quick and fair peer-review system, which is all easy to use. Visit http://www.dovepress.com/ testimonials.php to read real quotes from published authors. 